\title{
ESTUDIO RETROSPECTIVO DE LA EPIGLOTITIS AGUDA EN CANTABRIA EN EL PERIODO 1992-2015
}

\section{Retrospective study of acute epiglottitis in Cantabria in the period 1992-2015}

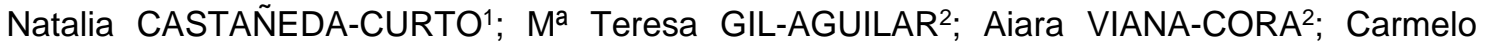 \\ MORALES-ANGULO²
}

\begin{abstract}
${ }^{1}$ Servicio de Otorrinolaringología. Hospital Comarcal de Laredo. Cantabria. España. ${ }^{2}$ Servicio de Otorrinolaringología. Hospital Universitario Marqués de Valdecilla. Santander. Cantabria. España
\end{abstract}

Correspondencia: nataliauc@gmail.com

Fecha de recepción: 30 de noviembre de 2017

Fecha de aceptación: 11 de diciembre de 2017

Fecha de publicación: 13 de diciembre de 2017

Fecha de publicación del fascículo: 1 de marzo de 2018

Conflicto de intereses: Los autores declaran no tener conflictos de intereses Imágenes: Los autores declaran haber obtenido las imágenes con el permiso de los pacientes Política de derechos y autoarchivo: se permite el autoarchivo de la versión post-print (SHERPA/RoMEO) Licencia CC BY-NC-ND. Licencia Creative Commons Atribución-NoComercial-SinDerivar 4.0 Internacional Universidad de Salamanca. Su comercialización está sujeta al permiso del editor adyacentes rápidamente progresiva que puede producir obstrucción severa de la vía aérea. Objetivo: conocer las características clínico-epidemiológicas de las epiglotitis aguda en Cantabria. Método: Estudio retrospectivo descriptivo de los pacientes diagnosticados de epiglotitis aguda desde enero de 1992 a abril de 2015 en la Comunidad Autónoma de Cantabria (España). Resultados: Cincuenta pacientes fueron incluidos en el estudio, con edades comprendidas entre 1 y 80 años, de los cuales cuarenta eran varones y diez eran mujeres. Sólo uno de ellos se trataba de un paciente pediátrico. Se diagnosticaron 2,7 casos por año, con una incidencia de 0,5 casos por 100000 habitantes y año. Dos pacientes tenían síndrome de Down. El síntoma principal de presentación fue odinofagia (88\%) seguido de disnea $(56 \%)$ y alteración en la voz (52\%). Nueve pacientes requirieron ingreso en cuidados Intensivos. Cuatro pacientes precisaron intubación orotraqueal; a cinco se les realizó una traqueotomía de urgencia. Siete pacientes presentaron complicaciones graves, uno de ellos con secuelas neurológicas severas. Ninguno de ellos murió a consecuencia de la epiglotitis. Conclusiones: La epiglotitis aguda es una patología poco frecuente pero grave y que, por tanto, precisa un tratamiento precoz para evitar complicaciones. Objetivamos una práctica desaparición de la epiglotitis en pacientes pediátricos en nuestro medio, lo que se explica por la introducción en el calendario vacunal de la inmunización contra el Haemophilus Influenzae.

PALABRAS CLAVE epiglotitis; Haemophilus influenzae; vacuna

SUMMARY Introduction and objective: Acute epiglottitis is an inflammation of the epiglottis and other adjacent structures that can produce severe obstruction of the airway. Our objective is to know the clinical-epidemiological characteristics of acute epiglottitis in a Region of the north of Spain. Method: Retrospective descriptive study of all patients diagnosed with acute epiglottitis from 
January 1992 to April 2015 in the Region of Cantabria (Spain). Results: Fifty patients were included in our study, aged between 1 and 80 years, of whom 40 were men and 10 were women. Only one of them was a pediatric patient. 2.7 cases / year were diagnosed, with an incidence of 0.5 cases per 100000 habitants per year. Two patients had Down syndrome. The main symptom was odynophagia (88\%) followed by dyspnea $(56 \%)$ and voice alteration $(52 \%)$. Nine patients required a stay in Intensive Care. Four patients required orotracheal intubation; five needed an emergency tracheotomy. Seven patients had serious complications, one of them with severe neurological damage. None of them died because of epiglottitis. Conclusions: Acute epiglottitis is a rare but serious disease and, therefore, requires early treatment to avoid complications. We have observed an almost total disappearance of epiglottitis in pediatric patients in our region, which is explained by the introduction in the vaccination calendar of immunization against Haemophilus influenzae.

epiglottitis; Haemophilus influenzae; vaccine

\section{INTRODUCCIÓN}

La epiglotitis aguda es una inflamación de la epiglotis y estructuras adyacentes rápidamente progresiva que puede producir obstrucción severa de la vía aérea y, en ocasiones, puede precisar intubación y/o traqueotomía urgente. Su incidencia en adultos es de 1 a 2 casos por 100.000 habitantes y año con una mortalidad que oscila entre 1 y $1,5 \%$ según las series [1]. Su principal agente causal era el Haemophilus influenzae tipo b (Hib) y se describía como una patología típica en niños de 2 a 6 años; sin embargo, tras la introducción de la vacuna contra este patógeno la incidencia en población pediátrica ha decrecido progresivamente y son otros gérmenes los responsables de la misma habitualmente [2, 3]. Clínicamente aparece odinofagia y disfagia acompañada o no por sialorrea. Además, pueden presentar fiebre, mal estado general o alteraciones en la voz. A medida que se produce obstrucción de vía aérea el paciente manifiesta disnea progresiva.

El objetivo de este trabajo es conocer las características clinicoepidemiológicas de la epiglotitis aguda en Cantabria.

\section{MATERIAL Y MÉTODO}

Se presenta un estudio retrospectivo descriptivo de los pacientes diagnosticados de epiglotitis en Cantabria, entre enero de 1992 y abril de 2015. Para la detección de casos se utilizó el registro del servicio de documentación clínica del Hospital Universitario Marqués de Valdecilla, usando los códigos CIE-9 MC (464.3). La muestra obtenida finalmente fue de $50 \mathrm{pa}-$ cientes (Tabla 1 y Figura 1). Se recogieron las siguientes variables: año de diagnóstico (Figura 2) clínica, técnica diagnóstica, microbiología, tratamiento, complicaciones y tiempo de estancia hospitalaria.
Tabla 1. Epiglotitis en Cantabria (periodo1992-2015). Características de la muestra.

\begin{tabular}{|lll|}
\hline Población total $(\mathrm{n})$ & & 50 \\
\hline Edad (años) & mínimo & 1 \\
& máximo & 80 \\
& media & 44,44 \\
\hline Sexo & Mujeres & 10 \\
& Hombres & 40 \\
\hline
\end{tabular}

\section{RESULTADOS}

El diagnóstico de epiglotitis aguda fue realizado a partir de los datos de la historia clínica y la exploración mediante laringoscopia indirecta o fibrolaringoscopia.

Los síntomas encontrados y las pruebas complementarias se encuentran reflejados en la Tabla 2. La odinofagia fue el síntoma más frecuente, estando presente en el $88 \%$ de los pacientes. Sólo un paciente presentó patología laríngea previa (quiste de epiglotis). Entre otros antecedentes personales hay que destacar que un paciente tuvo angina de Ludwig un mes antes, otro sufría una enfermedad de Crohn, un paciente presentaba serología positiva de virus de inmunodeficiencia humana y del virus de la hepatitis $C$ y dos pacientes tenían síndrome de Down.

Se tomó muestra para cultivo microbiológico en cuatro pacientes: en dos casos frotis faríngeos con resultado de flora mixta, un contenido de absceso epiglótico en el que se obtuvo $S$. milleri y en el paciente pediátrico un frotis con resultado Hib.

Todos fueron tratados con antibióticos sistémicos asociados o no a corticoides. El grupo antibiótico más utilizado fue cefalosporinas (55\%).

Nueve pacientes fueron ingresados en la unidad de cuidados intensivos (UCl). Cuatro pacientes precisaron intubación orotraqueal (uno 
de ellos fue el paciente pediátrico) y a cinco se les realizó una traqueotomía de urgencia.

En seis pacientes se objetivó presencia de un absceso epiglótico. Siete pacientes presentaron complicaciones graves: Dos presentaron shock séptico, uno infarto agudo de miocardio con derrame pleural bilateral, dos pacientes con una insuficiencia respiratoria con acidosis y otros dos sufrieron una parada cardiorrespiratoria.

Ningún paciente murió a consecuencia de la epiglotitis. Sin embargo, cabe destacar la evolución del paciente pediátrico. Tras una clínica de 12 horas de evolución de fiebre, irritabilidad y vómitos, presentó dificultad respiratoria de rápida instauración, llegando a urgencias pediátricas con parada respiratoria. Como consecuencia presentó encefalopatía posanóxica y como secuelas graves hipertonía con hipererreflexia y crisis comiciales.

El tiempo medio de estancia en el hospital fue de 8,76 días.

\section{DISCUSIÓN}

El organismo responsable del $75 \%$ al $90 \%$ de los casos de epiglotitis en la edad pediátrica era el Hib. Tras la introducción de la vacuna conjugada contra este germen a partir de 1987, la incidencia de enfermedades causadas por Hib se ha reducido drásticamente [2]. Esto ha hecho que actualmente sea más frecuente la epiglotitis en edades adultas y causada por otros patógenos.

Tabla 2. Epiglotitis en Cantabria (periodo1992-2015). Clínica y exploración.

\begin{tabular}{|l|r|r|}
\hline Síntomas & \multicolumn{1}{|c|}{$\mathrm{N}$} & \% (IC 95\%) \\
\hline Odinofagia & 44 & $88 \pm 9.0$ \\
\hline Disnea & 28 & $56 \pm 13.7$ \\
\hline Alteraciones de voz & 26 & $52 \pm 13.8$ \\
\hline Fiebre & 23 & $46 \pm 13.8$ \\
\hline Disfagia & 22 & $44 \pm 13.7$ \\
\hline Sialorrea & 5 & $10 \pm 8.3$ \\
\hline \multicolumn{2}{|r|}{} \\
\hline Diagnóstico indi- & 31 & $62 \pm 13.4$ \\
\hline $\begin{array}{l}\text { Laringoscopia } \\
\text { recta }\end{array}$ & 18 & $36 \pm 13.3$ \\
\hline Fibrolaringoscopia & $10 \pm 8.3$ \\
\hline Rx cervical & 5 & $18 \pm 10.6$ \\
\hline TAC
\end{tabular}

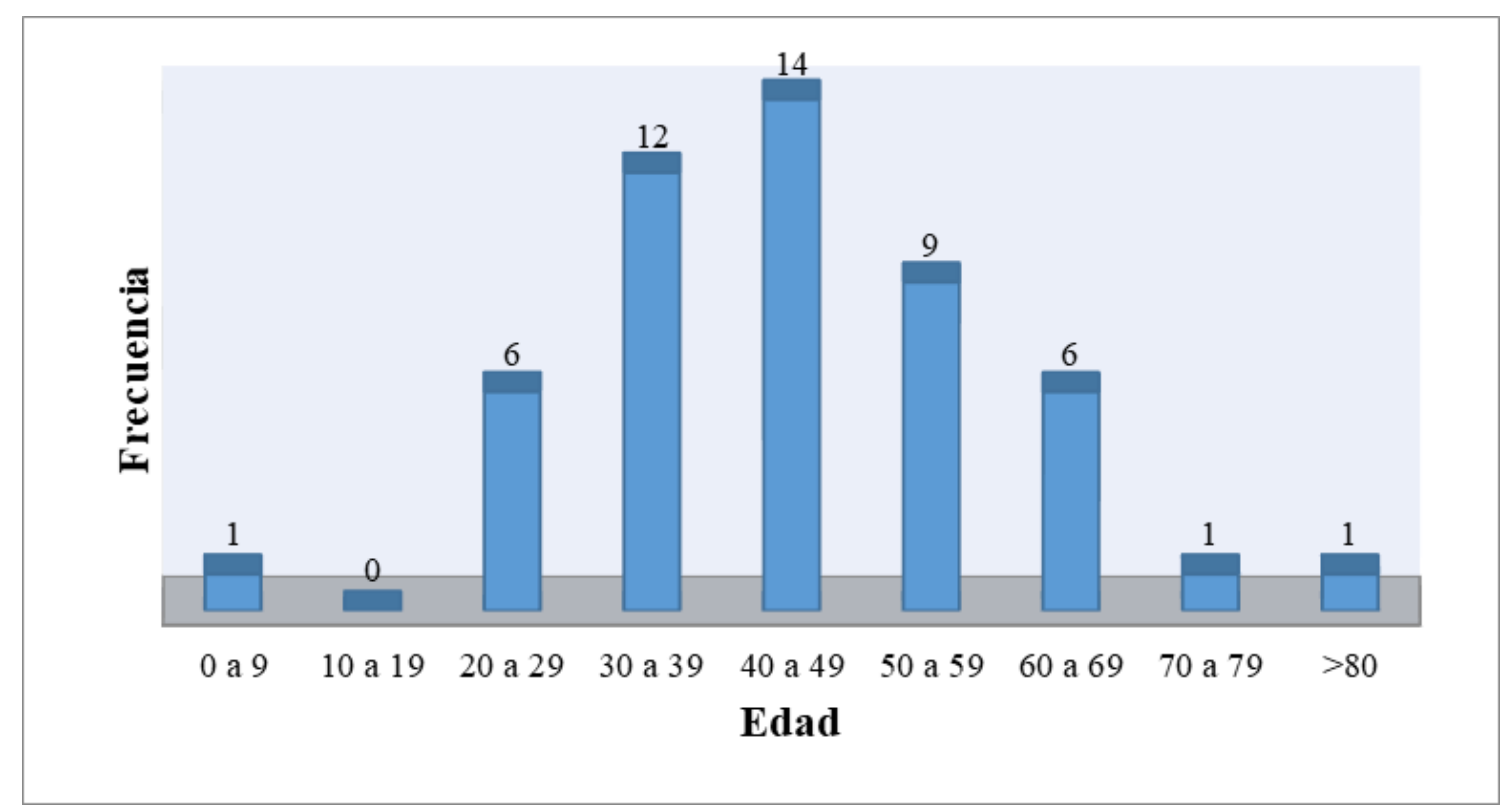

Figura 1. Epiglotitis en Cantabria (periodo1992-2015). Distribución de casos por franjas de edad. 


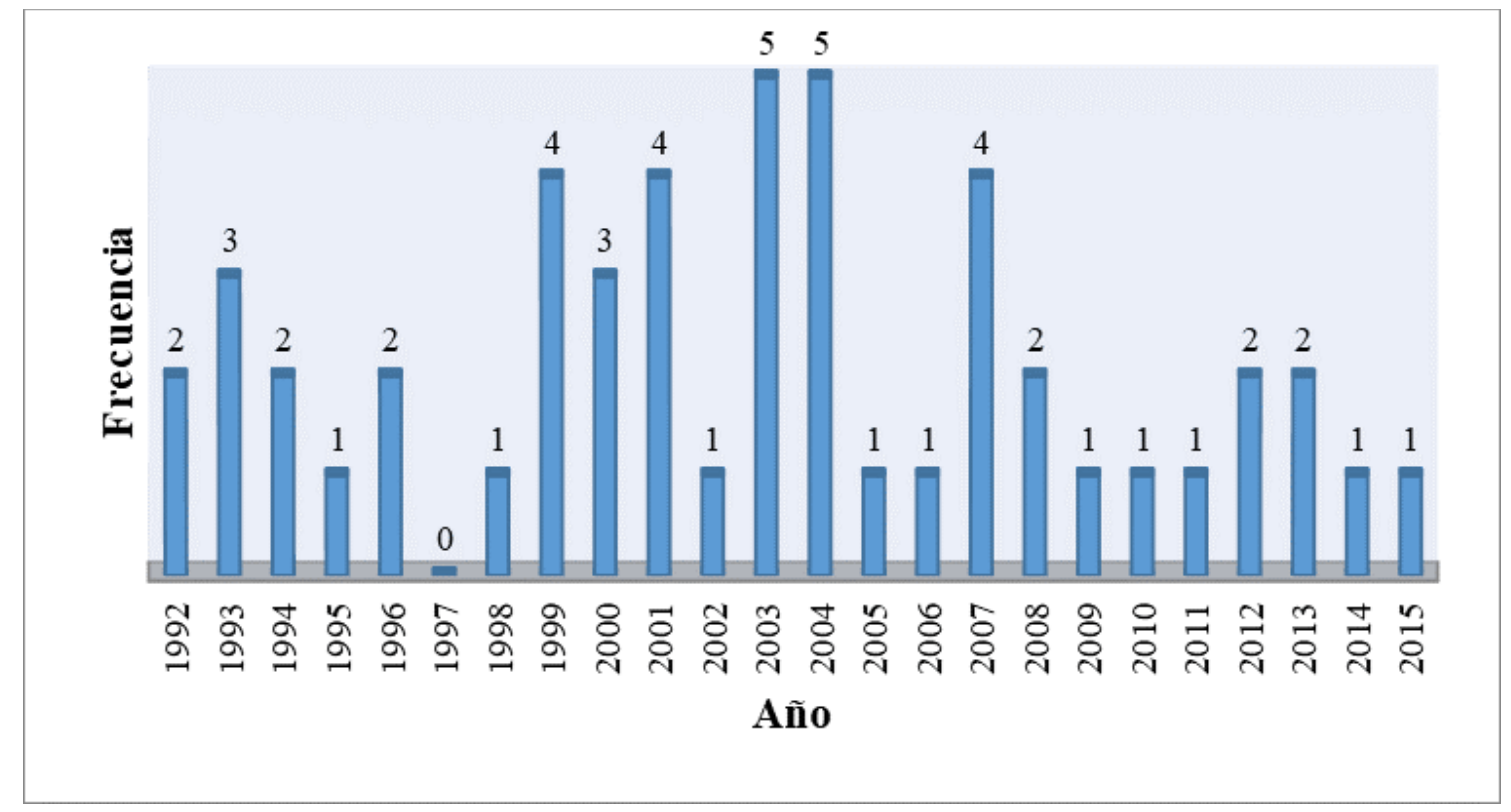

Figura 2. Epiglotitis en Cantabria (periodo1992-2015). Distribución de casos por año.

En nuestra serie tan solo había un paciente pediátrico y se produjeron 2,7 casos / año, con una incidencia de 0,5 casos por 100000 habitantes y año. Al compararlo con otros estudios objetivamos algunas diferencias. Briem B y cols en su estudio en Islandia, objetivan una incidencia de 1,4 casos por 100000 habitantes y año antes del inicio de la vacuna y sin embargo una incidencia de 0,77 casos por 100000 habitantes y año en los años posvacuna, lo que se aproxima más a nuestros datos. En otro estudio realizado en Dinamarca [3] describen la incidencia en adultos en 1,9 casos por 100000 habitantes y año lo cual es bastante superior a nuestros datos. Así mismo revisando un estudio realizado en Extremadura [1] obtenían un total de 2 casos por año, siendo nuestra cifra levemente superior.

Por otra parte, se muestra un claro predominio de hombres (80\%), al igual que en otras publicaciones $[1,5,6]$.

Llama la atención en nuestra serie que dos pacientes tenían síndrome de Down, ya que sólo hemos encontrado un caso previamente publicado en la literatura médica [7].

La mayor parte de los pacientes consultaron por odinofagia $[1,5,6]$, acompañada o no de fiebre, disfagia y/o sialorrea. Esta forma de presentación difiere de la clásica descrita en niños, en los que la disnea es el síntoma principal con sialorrea y la «posición de trípode».
A todos los pacientes se les realizó exploración laríngea de manera directa o indirecta. En adultos no hay contraindicación para realizar una laringoscopia cuidadosa. Sin embargo, en pacientes pediátricos no está recomendado ya que, al ser una vía aérea más estrecha, hay mayor riesgo de obstrucción aguda [1]. Nuestro paciente pediátrico fue diagnosticado de epiglotitis mediante exploración directa al intentar intubarle por parada respiratoria. Además de visión directa, puede contribuir al diagnóstico la observación de «signo del pulgar» en la radiografía simple, que es la visión lateral de la epiglotis edematizada [8]. En nuestro estudio fue infrecuente la realización de TAC que generalmente se utiliza para el diagnóstico de complicaciones derivadas.

En cuanto al estudio microbiológico, Briem B y cols obtienen un cultivo positivo para Hib en el $50 \%$ de los pacientes, lo cual es posiblemente debido a que la serie incluye casos anteriores al inicio de la vacunación, un $35 \%$ es positivo para estreptococos y un $15 \%$ para otros organismos.

El tratamiento antibiótico intravenoso asociado a corticoides debe ser iniciado lo antes posible de manera empírica dado el alto riesgo de obstrucción de vía aérea. Al igual que en otras series [5], las penicilinas fueron el tratamiento de elección en la mayor parte de los casos, siendo el $55 \%$ cefalosporinas. 
Las complicaciones de la epiglotitis pueden ser graves. Aunque en nuestra serie no falleció ningún paciente, siete de ellos evolucionaron desfavorablemente con complicaciones serias (shock séptico, infarto agudo de miocardio, insuficiencia respiratoria) produciéndose en dos pacientes una parada cardiorrespiratoria. Además, cabe destacar al único paciente pediátrico registrado, que tuvo secuelas neurológicas severas por hipoxia a consecuencia de la disnea aguda producida por la epiglotitis. Otras complicaciones registradas en la literatura son neumonía o mediastinitis. [1, 5]

La obstrucción de la vía aérea puede requerir manejo invasivo con intubación orotraqueal o traqueotomía urgente. En nuestra población estudiada, estos eventos se produjeron en un $8 \%$ y $10 \%$ respectivamente, cifras similares a las obtenidas en otros estudios en adultos [1]. Algunos autores se muestran a favor de ingreso sistemático en UCI frente a otros que prefieren un seguimiento más conservador [6]. De una forma u otra, cabe resaltar la necesidad de ingresar al paciente en un hospital que disponga de UCI.

\section{CONCLUSIONES}

La epiglotitis aguda es una patología poco frecuente pero grave y que, por tanto, precisa un manejo precoz para evitar complicaciones. Ante su sospecha es importante un diagnóstico rápido, la instauración de antibióticos sistémicos empíricos de forma inmediata y el ingreso en un hospital que cuente con una UCI dado el alto riesgo de obstrucción de vía aérea superior

Gracias a la vacuna contra Hib la epiglotitis en población infantil prácticamente ha desaparecido en nuestro medio.

\section{BIBLIOGRAFÍA}

1. Pino Rivero V, González Palomino A, Pantoja Hernández CG, Mora Santos ME, Gil Calero M, Rodríguez Carmona $\mathrm{M}$, et al. Epiglotitis aguda en adultos. Nuestra experiencia. Acta Otorrinolaringol Esp. 2007;58(6):263-5.

2. Sakar S, Roychoudhury A, Roychoudhury B K. Acute epiglotitis in adults - a recent review in an Indian Hospital. Indian J Otolaryngol Head and Neck Surg. 2009;61:197-9.

3. Guldfred L, Lyhne D, Becke, B. Acute epiglotitis: Epidemiology, clinical presentation, management and outcome. J Laryngol Otol. 2008;122(8):818-23.

4. Shah RK, Roberson DW, Jones DT. Epiglottitis in the Hemophilus Influenzae Type B Vaccine Era: Changing Trends. Laryngoscope 2004;114:557-60.

5. Qazi I M, Jafar A M, Hadi KA, Hussain Z. Acute epiglottitis: a retrospective review of 47 patients in Kuwait. Indian J Otolaryngol Head Neck Surg. 2009;61: 301-5.

6. Briem B, Thorvardsson $\mathrm{O}$, Petersen $\mathrm{H}$. Acute epiglotitis in Iceland 1983-2005. Auris Nasus Larynx 2009;36:46-52.

7. Imataka $\mathrm{G}$, Mitsui $\mathrm{M}$, Mitsui $\mathrm{N}$, Hirabayashi $\mathrm{H}$, Yamanouchi $\mathrm{H}$ and Eguchi M. Down syndrome with acute epiglotitis. Pediatr Int. 2005;47:333-5.

8. Angirekula V, Multani A. Epiglottits in an adult. N Engl J Med 2015;372:e20. 